\title{
Blindness and scalp haematoma in a child following a snakebite
}

\author{
Oludolapo Sherifat Katibi ${ }^{1}$, Feyiyemi Grace Adepoju ${ }^{2,4}$, Benedict Oluwasesan Olorunsola ${ }^{3}$, \\ Samuel Kolade Ernest ${ }^{1,3}$, Kehinde Fasasi Monsudi ${ }^{5}$
}

1. Department of Paediatrics and Child Health, College of Health Sciences, University of Ilorin, Ilorin, Kwara State, Nigeria.

2. Department of Ophthalmology, College of health Sciences, University of Ilorin, Ilorin, Kwara state, Nigeria.

3. Department of Paediatrics and Child Health, University of Ilorin Teaching Hospital, Ilorin, Kwara State, Nigeria.

4. Department of Ophthalmology, University of Ilorin Teaching Hospital, Ilorin, Kwara state, Nigeria.

5. Federal Medical Centre Birnin Kebbi, Kebbi state, Nigeria. .

\begin{abstract}
Background: Snake envenomation is a major public health problem of the Savannah regions of West Africa. Ocular manifestations of snakebites are rare with few reports documenting blindness as a complication.

Objective: To highlight an unusual manifestation of snake bites and its attendant problems

Methods: A report of scalp haematoma and blindness in a 10 year old child presenting 2 weeks after a snake bite (presumably carpet viper) is a rare manifestation. Local swelling, epistaxis, bilateral proptosis, exposure keratopathy and use of traditional eye medications were associated findings. Anti-venom though administered late saved the child's life but blindness could not be reversed.

Results: Ocular ultrasonography revealed layered retrobulbar collection in the left eye, presumably due to hemorrhage. The skull x-ray showed a soft tissue swelling and aspirate from scalp swelling was bloody. Cranial Computed Tomography (CT) scan done late detected no abnormalities.

Conclusion: Snakebite is associated with lifelong morbidity. Ocular manifestations must be treated as emergency. This case highlights the effect of ignorance and poverty in a setting of a common medical emergency leading to blindness and reduced quality of life.
\end{abstract}

Keywords: snakebite, blindness, scalp haematoma, child

DOI: http://dx.doi.org/10.4314/ahs.v15i3.46

Cite as: Katibi OS, Adepoju FG, Olorunsola BO, Ernest SK, Monsudi KF. Blindness and scalp haematoma in a child following a snakebite. Afri Health Sci. 2015;15(3):1041-4. doi: http:/ / dx.doi.org/10.4314/ahs.v15i3.46

\section{Introduction}

Snake envenomation is a major public health problem of the Savannah regions of West Africa. Morbidity and mortality is high due to poor health facilities, ignorance, poverty and inadequate access to anti-venom. In sub-Saharan Africa, 314,000 bites and 7,300 deaths occur annually. ${ }^{1}$ Nigeria has one fifth of all the cases occurring in the region with $10 \%$ of ward admissions in the Savannah region attributable to snake bites. ${ }^{2}$ Oc-

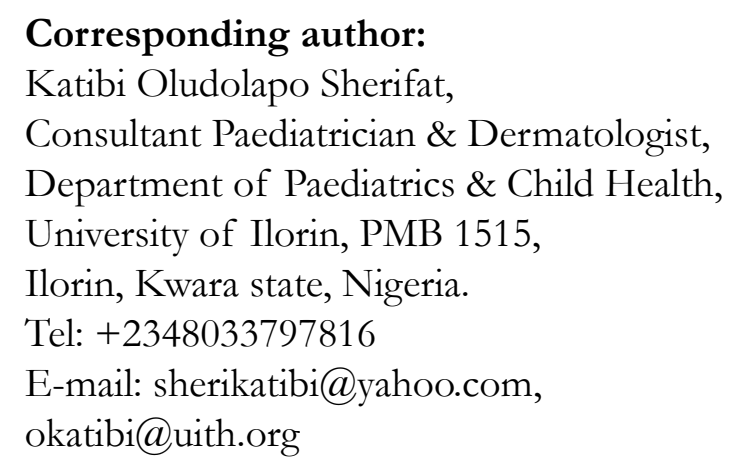

ular complications following snake envenomation are relatively rare. Some studies looking at the varied presentations of snake bites did not report any ocular manifestations. ${ }^{3,4}$ However, a few cases of ocular problems caused by neurotoxic cobras or haemotoxic vipers have been documented in some parts of the world., ${ }^{5,6}$ We report a case of bilateral blindness in a 10 year old boy following a viper bite and to the authors' knowledge, the first in Southern Nigeria.

\section{Case report}

A 10 year old boy from a rural community in South west, Nigeria presented at the Emergency Paediatric unit with a 2 week history of swelling of the left middle finger following a bite by a brownish snake. The snake was presumed to be a viper from description, though the snake escaped. The swelling extended to the whole left upper limb within a few days. He had minor bleed- 
ing from the nose with no obvious bleeding from any other site of the body. Bilateral proptosis was noticed a week after with associated itching, tearing, foreign body sensation and loss of vision (Fig. 1).

Fig 1. Bilateral proptosis with chemosis at presentation

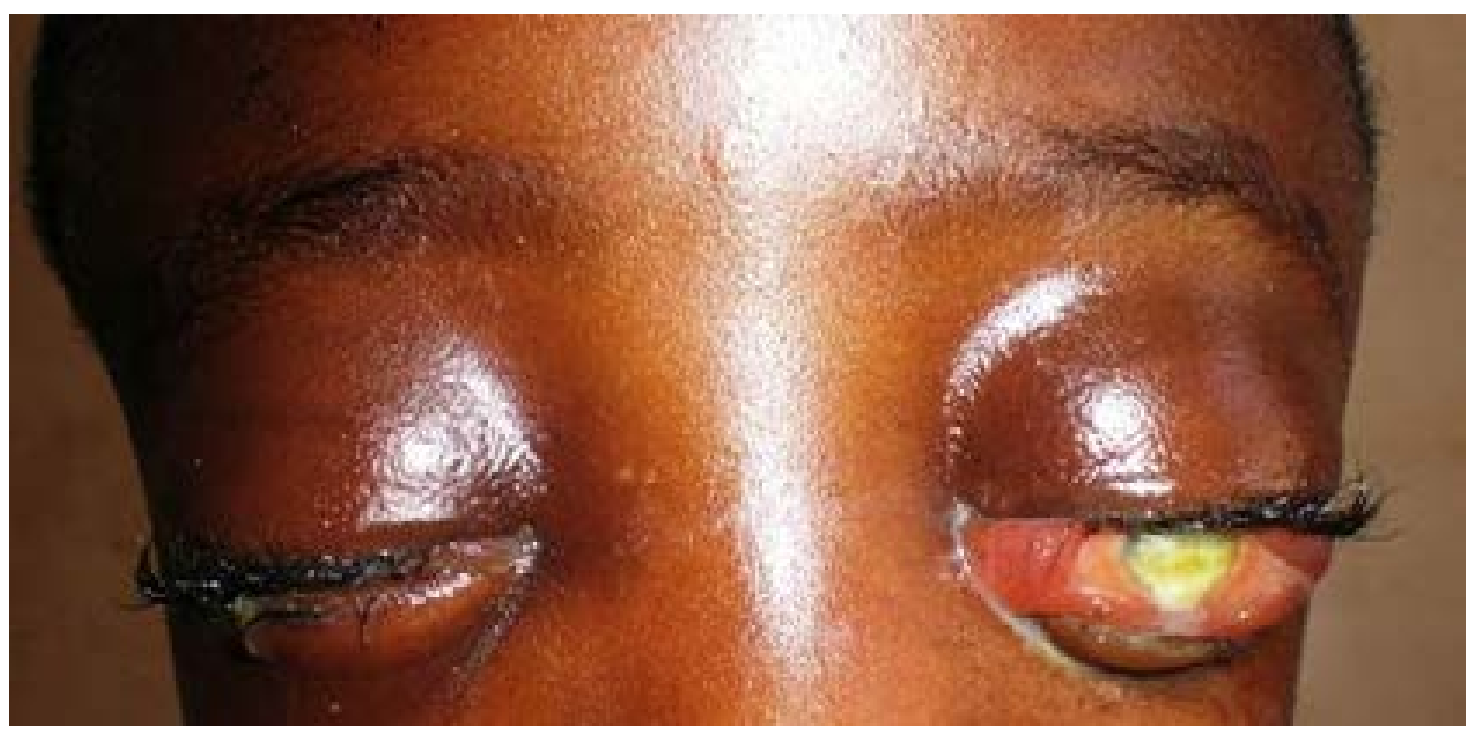

He also had progressive head enlargement and generalized headache. There was no history of vomiting, nausea, convulsions or loss of consciousness. He was treated by traditional healers with application of herbal concoction to the eyes and orally with no significant improvement.

Examination revealed an alert and well oriented child with obvious macrocephaly (occipito-frontal circumference of $58 \mathrm{~cm}$ ) and a boggy scalp swelling extending from the frontal to the parietal area just short of the occiput. Swelling was fluctuant with no differential warmth and no tenderness. He was pale (PCV 18\%) and tachycardic though blood pressure was normal. There was no significant lymph node enlargement and no petechiae hemorrhages nor purpura.

The left middle finger was hyperpigmented and necrotic with neither loss of sensation nor limitation of movement (Fig. 2) Visual acuity in both eyes was no perception of light, and bilateral non-axial proptosis, worse on the left ( RE 26mm, LE $28 \mathrm{~mm}$ ) with severe conjunctival chemosis. There was corneal haziness bilaterally from exposure keratopathy and possibly traditional eye medications with no view of further details.

Figure 2: Site of bite: Left middle finger which is swollen, hyper pigmented with skin necrosis

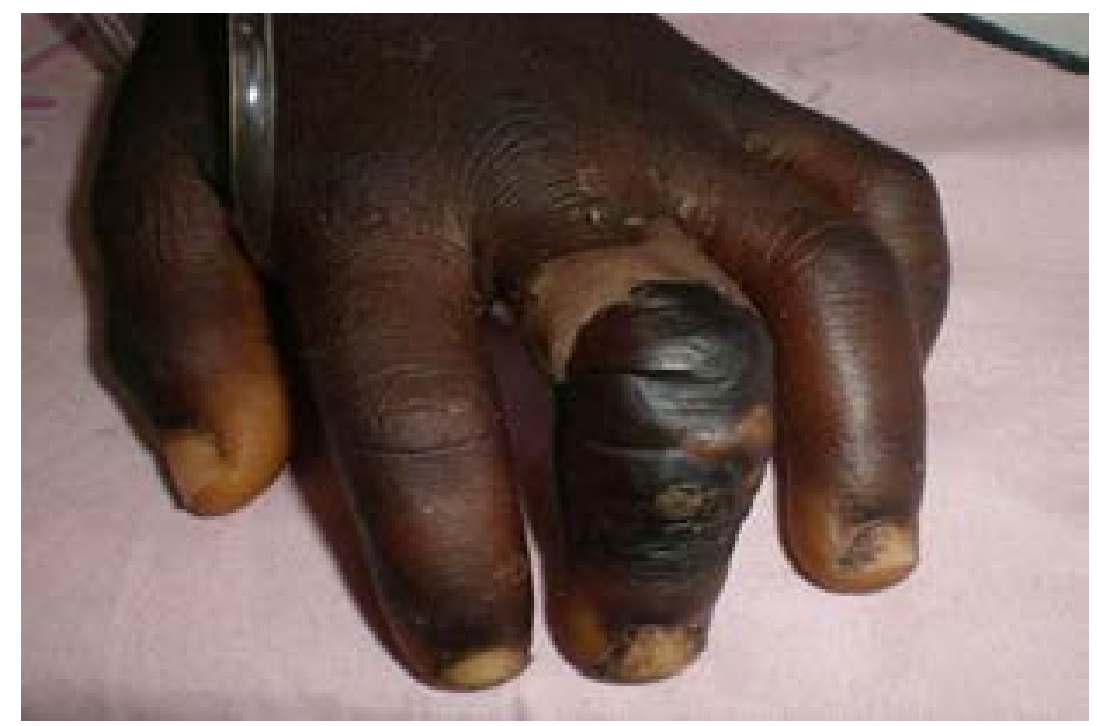


He also had bilateral lid oedema with complete ophthalmoplegia. He was managed for grade 3 systemic envenomation of a snakebite with a scalp haematoma, bilateral blindness and a left mid finger cellulitis.

The skull x-ray showed a soft tissue swelling in keeping with a scalp haematoma. Radiograph of the left mid finger and the clotting profile were normal. Aspirate from the scalp was bloody and microscopy and culture yielded no growth. Ocular ultrasonography revealed layered

Fig. 3: Bilateral corneal opacities and blindness

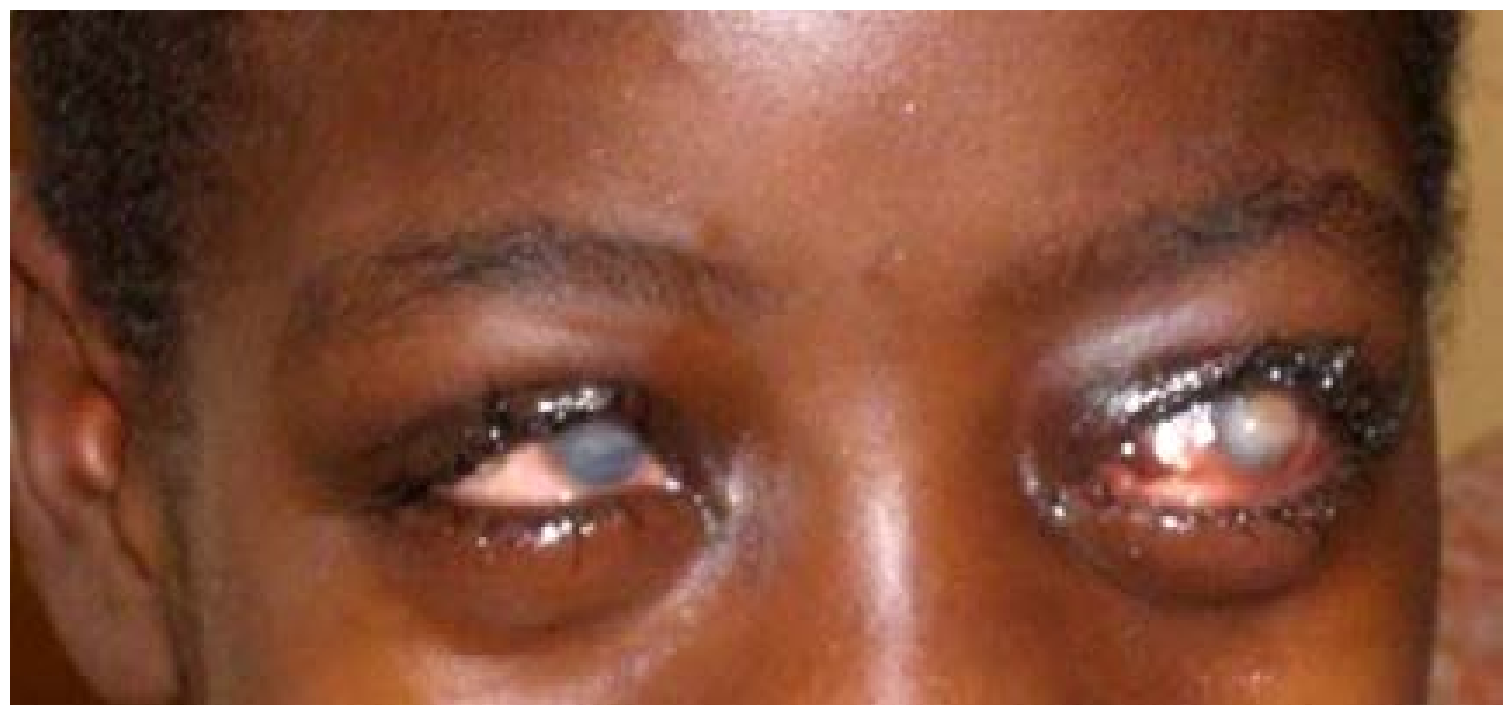

retrobulbar collection in the left eye, presumably due to hemorrhage. Cranial Computed Tomography (CT) scan to detect intracranial bleeds was done much later when symptoms had resolved due to non-availability of funds. By that time, no abnormalities were detected.

Treatment was commenced with transfusion of two pints of blood and administration of polyvalent anti-venom, anti-tetanus toxoid, intravenous antibiotics, analgesics, copious eye ointment, topical antibiotics and oral acetazolamide. A tarsorraphy was initially planned for but due to financial and logistic difficulties, it was eventually not done.
There was gradual reduction in the scalp swelling and proptosis, but minimal improvement in the eye sight with bilateral corneal opacities (Fig 3). Child was discharged after one month of admission.

\section{Discussion}

The carpet viper (Echis Ocellatus), black-necked spitting cobra (Naja nigricolis) and the puff adder (Biti arietans) are the commonest species of venomous snakes seen in Nigeria. However, the carpet viper described as the most dangerous snake in the world, is the major cause of morbidity and mortality in the country. It accounts for $90 \%$ of bites and $60 \%$ of the fatalities in the country. ${ }^{7}$

Viper snakebites are typically associated with marked haemorrhage, local swelling and necrosis. ${ }^{8}$ All of these occurred in this boy which supported presumption of the viper as the culprit snake. The pro-coagulant enzymes in the venom of the carpet viper activate the 
Visual loss in this patient can be attributed to increased intraorbital pressure from retrobulbar hemorrhage, compression of/ or compromise to the blood supply of the optic nerve and optic neuritis because of poor pupillary reactivity (though fundus could not be visualised). Orbital infection (cellulitis), exposure keratopathy and application of offensive traditional eye medication from the herbal doctor were other contributors to this visual loss.

Neuroimaging (especially Magnetic Resonance Imaging (MRI) would have been useful in the immediate period not only to rule out intracranial haemorrhage but also to better visualize the orbits. It is particularly important in differentiating neuropathy from infarction because of good visualization of cranial and intraorbital nerves. ${ }^{14}$ However, MRI was unavailable for this patient.

This report has highlighted the magnitude of problems associated with snake envenomation in Nigeria. Ignorance, poverty, cultural practices as well as a poor health system have contributed to this morbidity. Most individuals have to pay out of pocket for health services even in government owned public hospitals as seen in this case. Also, patients who are at most risk (youth, farmers, rural dwellers) are less likely to afford these health care services leading them to approach alternative medical practitioners who are cheaper. Apart from tackling these contributors to morbidity, complications of snake bite can be mitigated by using the haddon matrix where the host (affected individual), the agent (snake) and the environment are considered in the following ways. ${ }^{15}$

Pre-event: Health education to correct myths that snakebites are innocuous and can be well managed with traditional medications, plugging of holes and avoidance of hidden crevices, use of protective footwear, practice of mechanised farming and extra vigilance in favourable seasons like the summer.

Event: Avoidance of deliberate provocation of snakes, immediate transfer to health care facilities for first aid, discouragement of harmful practices like local incisions, sucking of wound site and tourniquet application.

Post-event: Ready availability of anti-snake venom, strengthened health system with prompt and efficient referral and emergency services with free or subsidized health care especially for life threatening situations.

\section{Conclusion}

This case illustrates the lifelong morbidity that may arise following snakebites if not properly managed. Ocular involvement following snakebites though rare, is an emergency which requires immediate attention to prevent blindness. Late presentation, traditional eye medication and poverty hampering investigations and treatment may have worsened prognosis. Though the anti-snake venom administered late saved the boy's life, he went home blind in both eyes.

\section{Conflict of interest:}

None

\section{References}

1. Chippaux JP: Estimate of the burden of snakebites in sub-Saharan Africa: a meta-analytic approach. Toxicon 2011, 57(4):586 PubMed -599. Doi: 10.1016/j. toxicon. 2010.12.022.

2. Habib AG. Public health aspects of snakebite care in West Africa : perspectives from Nigeria. J Venom Anim Toxins incl Trop Dis. 2013, 19(1):27.

3. Hayat AS, Khan AH, Shaikh TZ, Ghouri RA, Shaikh N. Study of snake bite cases at Liaquat University Hospital Hyderabad/Jamshoro. J Ayub Med Coll Abbottabad 2008, 20:125-127.

4. Warell DA, Davidson NMcD, Greenwood BM, et al. Poisoning by bites of the saw-scaled or carpet viper in Nigeria. QJ Med 1977, 46:33- PubMed ,62.

5. Kweon E, Lee D, Ahn M, Nork T, Cho N. Vision loss following snakebite in a patient with controlled Aplastic anaemia. J Venom Anim Toxins incl Trop Dis. 2009, 15:163-167.

6. Rao KV. Optic neuritis and ophthalmoplegia caused by snake bite. Indian J Ophthalmol 1981, 29:243-245

7. Paramonte B. Snake bites in Nigeria. Med J Ther. 2007, 1:222- PubMed ,226. .

8. Warrell DA, Arnett C: The importance of bites by the saw-scaled or carpet viper (Echis carinatus): epidemiological studies in Nigeria and a review of the world literature. Acta Trop 1976, 33(4):307 PubMed -341.

9. WHO. Guidelines for the Prevention and clinical management of Snakebite in Africa. Mauritius: WHO regional office, Africa, 2010. .

10. Rao BM. A case of bilateral vitreous haemorrhage following snake bite. Indian J Ophthalmol 1977, 25:1-2.

11. Berger RR, Brook S.Cobrabite:Ophthalmic manifestaions. Harefuah 1993, 125:265-266 PubMed 12. Mustapha SK, Mubi BM, Askira BH. Bilateral blindness following snakebite. Trop Doct. 2010,40:117-118.

13. Guttmann-Friedmann A. Blindness after snake bite. Brit J Ophthal. 1956,40:57- PubMed ,59.

14. Kakaria AK. Imaging in neuro-ophthalmology: An overview. Oman J Ophthalmol 2009, 2: 57-61 PubMed . 15. Barnett DJ, Balicer RD, Blodgett D, et al. The application of the Haddon matrix to public health readiness and response planning. Environ Health Perspect 2005, 113: 561-566. 